\title{
Effects of Explosion Shock Waves on Lung Injuries in Rabbits
}

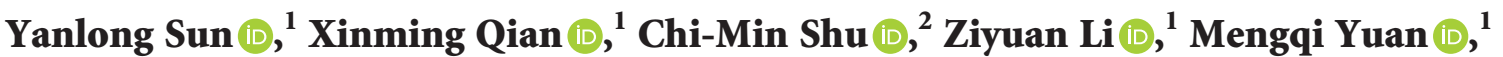 \\ Qi Zhang $\left(\mathbb{D},{ }^{1}\right.$ and Yanteng $\mathrm{Li} \mathbb{1}^{3}$ \\ ${ }^{1}$ State Key Laboratory of Explosion Science and Technology, Beijing Institute of Technology, Beijing, China \\ ${ }^{2}$ Department of Safety, Health, and Environmental Engineering, National Yunlin University of Science and Technology, Douliu, \\ Yunlin, Taiwan \\ ${ }^{3}$ Department of Neurosurgery, Navy General Hospital of PLA, Beijing, China
}

Correspondence should be addressed to Mengqi Yuan; myuan@bit.edu.cn

Received 15 November 2020; Revised 29 December 2020; Accepted 31 January 2021; Published 10 February 2021

Academic Editor: Isabelle Sochet

Copyright (C) 2021 Yanlong Sun et al. This is an open access article distributed under the Creative Commons Attribution License, which permits unrestricted use, distribution, and reproduction in any medium, provided the original work is properly cited.

\begin{abstract}
The purpose of this study was to explore the damage effects and injury mechanism of free-field explosion shock waves on rabbit lungs. Six free-field explosion experiments, each with $500 \mathrm{~g}$ trinitrotoluene (TNT), were conducted as the shock wave overpressure acting on the rabbits was measured. The peak overpressure of the shock wave was 533,390,249, 102, and $69 \mathrm{kPa}$ at the respective test points. Damage to the rabbit lungs caused by shock wave overpressure was investigated through observation, anatomical analysis, and hematoxylin-eosin (HE) staining processing. The shock wave overpressure of 69-102 kPa caused mild-to-moderate injury; the shock wave overpressure of 102-249 kPa caused moderate injury; the shock wave overpressure of 249-390 kPa resulted in moderate-to-severe injury; and the shock wave overpressure of $390-533 \mathrm{kPa}$ caused severe injury to the rabbit. Mild, moderate, and severe injuries destroyed some, most, or all alveolar structures, correspondingly, as well as producing partial cell apoptosis. The overpressure damage mechanism primarily involves the collapse and rupture of pulmonary alveolus in the lung tissue. As a novel attempt, the investigation provided here may serve to improve the current shock wave injury mechanism.
\end{abstract}

\section{Introduction}

Explosive weapons may severely injure civilians as well as state officials during military conflicts and terrorist attacks [1-7]. Injury to the lung as induced by the weapons is one of the most dangerous, and even fatal, results of an explosion. The lung, as a gas-containing organ, is highly vulnerable to explosive overpressure and particularly susceptible to barotrauma [8-10]. The incidence of primary blast lung injury (PBLI) in immediate fatalities may be as high as $47 \%$ [11]. In a study on explosive injury victims who survived to emergency admission, PBLI was present in $11.2 \%$ of $648,16.2 \%$ of mounted injuries, and $17.1 \%$ of dismounted injuries in the same sample developed for PBLI, which is significantly associated with increased mortality $[12,13]$. The main physiological characteristics of PBLI are pulmonary hemorrhage, edema, and microcirculation dysfunction, alveolar rupture, pulmonary bullae, and atelectasis; any or all of these may be present at varying extent of severity.
Form literature, previous researchers had explored lung injury effects and explosion shock wave mechanisms. Research showed that the functional and morphological damage to animals is most severe in combined injury groups. A high velocity fragment striking the extremity aggravates blast injury to the lungs. The most commonly injured organ in an explosion is indeed the lungs, which may be unaccompanied by any aggravation to the heart or abdominal organs [14]. Explosive experiments conducted on sheep have shown that the lung is most sensitive to trinitrotoluene (TNT) explosions, whereas the upper respiratory tract is most sensitive to muzzle explosion waves. The injury thresholds of overpressure were 29.0, 29.5, and $41.2 \mathrm{kPa}$ for the upper respiratory tract, lungs, and gastrointestinal tract, respectively, at a single exposure. Repeated exposure (up to 60 explosions) also reduced the injury threshold of the internal organs. Existing safety limits protect $90 \%$ of the exposed population against internal organ injuries due to weak explosion shock waves [15]. 
Lung injury severity increases as the peak pressure and duration of an explosion increases. This relationship can be expressed with linear regression equations; the physical parameters of the underpressure can be used to indicate the severity of the injury to the lungs, as well [8]. Previous researchers had determined the threshold range of damage to animals under the action of two types of complex waves generated by TNT explosions [16]. The damage effects of blast overpressure and underpressure on the lungs of rats and rabbits were investigated with a self-made shock wave segmented simulator, for example, to reveal the mechanism of overtension effects in blast injuries [17].

There is a wealth of extant research on the biological effects of overpressure on the lungs [18-20]. However, the precise mechanism of injury is not known yet. In this study, we examined the damage effects and injury mechanisms of TNT explosion shock waves on the lungs of rabbits to explore the relationship between the physical parameters of the waves and internal organ injury. This analysis may serve to define effective safety limits for weak blast waves as well as safety limits on battle training for military personnel; our results may also be used to design weak blast wave protection devices.

\section{Materials and Methods}

2.1. Animals. A total of 32 adult male rabbits, each weighing $2.0-2.5 \mathrm{~kg}$, were used in the experiment. Thirty of them were anesthetized using $1.5 \%$ pentobarbital sodium in a dose of $30 \mathrm{mg} / \mathrm{kg}$ per body weight and morphine $(5 \mathrm{mg} / \mathrm{kg}$ ) was used to relieve their pain. We divided them into six groups of five rabbits each. The remaining two (without anesthesia) were used as a control group. Procedures involving animals and their care were conducted in conformity with NIH guidelines (NIH Pub. No. 85-23, revised 1996) and were approved by Institutional Animal Care and Use Committee of the Navy General Hospital of PLA (People's Liberation Army), China.

2.2. Overpressure Calculation. As planned, we estimated the peak overpressure of shock wave in a $500 \mathrm{~g}$ TNT explosion at different locations based on the empirical formula established by Sadovskyi. The calculated values were used to determine the distances of the rabbits from the center of the explosion. The target shock wave overpressure range was set to $75-550 \mathrm{kPa}$. Location distances were selected as $1.0,1.2$, $1.5,2.0$, and 2.5 metres $(\mathrm{m})$, accordingly.

Sadovskyi's empirical formula [21] is expressed as in the two following equations:

$$
\begin{aligned}
& \Delta p_{1}=\frac{1.07}{\bar{r}^{3}}-0.1, \quad \bar{r} \ll 1.0, \\
& \Delta p_{1}=\frac{0.076}{\bar{r}}+\frac{0.255}{\bar{r}^{2}}+\frac{0.65}{\bar{r}^{3}}, \quad 1.0 \ll \bar{r} \ll 15,
\end{aligned}
$$

where $\bar{r}=r / \sqrt[3]{w}$ is the "contrast distance," $w$ is the TNT equivalence, $r$ is the distance from the center of the explosion to the test point, and $\Delta p_{1}$ is the peak overpressure of the blast shock wave. Units are $\mathrm{kg}, \mathrm{m}$, and $\mathrm{MPa}$, respectively.
2.3. Experimental Setup. Tests were conducted at the East Garden Experimental Base of Beijing Institute of Technology, which was designed specifically for explosion experiments. The layout of the experimental site is shown in Figure 1 and Supplementary Figure 1.

The explosion test system primarily consists of cylindrical TNT explosives, electric detonators, an initiator, trigger lines, pressure sensors, a multichannel transient recorder, and a high-speed camera. The connections among test parts are shown in Supplementary Figure 2. The distances between rabbits and the explosion center were 1.0, $1.2,1.5,2.0$, and $2.5 \mathrm{~m}$, individually. The TNT, pressure sensors, and rabbits were all placed $1.5 \mathrm{~m}$ above the ground. Free-field pressure sensors were used to measure the overpressure of each explosion shock wave. The TNT explosives were detonated by electric detonators as experimental data were gleaned and recorded by the multichannel transient recorder. The free-field explosion experiments were carried out in six replications each with $500 \mathrm{~g}$ TNT. The peak overpressures at the test points in all six explosions were determined by analyzing these data.

Prior to the test, the rabbits were anesthetized and fixed on brackets with ligaments so that their chests and abdomens faced the explosion center. Pressure sensors were properly fixed beside the rabbits. Sensors from the inside to the outside of the explosion center were, respectively, labeled as channels (ch) 1 , ch 2, ch 3, ch 4, and ch 5, as shown in Figure 1. The high-speed camera was initiated at the moment the TNT was detonated to record the explosion process. The rabbits were removed from the brackets just after the experiment was completed. Injury to their lungs was judged by preliminary observation; then the rabbits were dissected to further observe the injuries. The lungs were then removed from the thorax, examined, photographed, and immersed in 10\% formalin. The fixed lungs were sectioned, embedded with paraffin, and examined microscopically to observe histopathological changes. The animal's anatomy and naked eye observation in the explosion field and the subsequent section production and treatment were all completed by medical chairs doctors of the Navy PLA at China's General Hospital, Beijing.

2.4. Statistical Analysis. The explosion experiment was repeated 6 times. SPSS 24.0 software (IBM Corp., Armonk, NY, USA) was used for statistical analysis of experimental data. Comparisons of means among and within groups were performed using one-way repeated-measures analysis of variance (ANOVA). Differences in count data were tested for statistical significance with the Chi-square test. A $P$ value $<0.05$ was considered statistically significant.

\section{Results}

3.1. Computational Simulation. A simulation model in ANSYS LS-DYNA was built to determine the relation between the TNT equivalent and distance from the explosion center based on the experimental scenario (Figure 2). The shock wave overpressures at the test points were calculated to glean the results discussed below. 


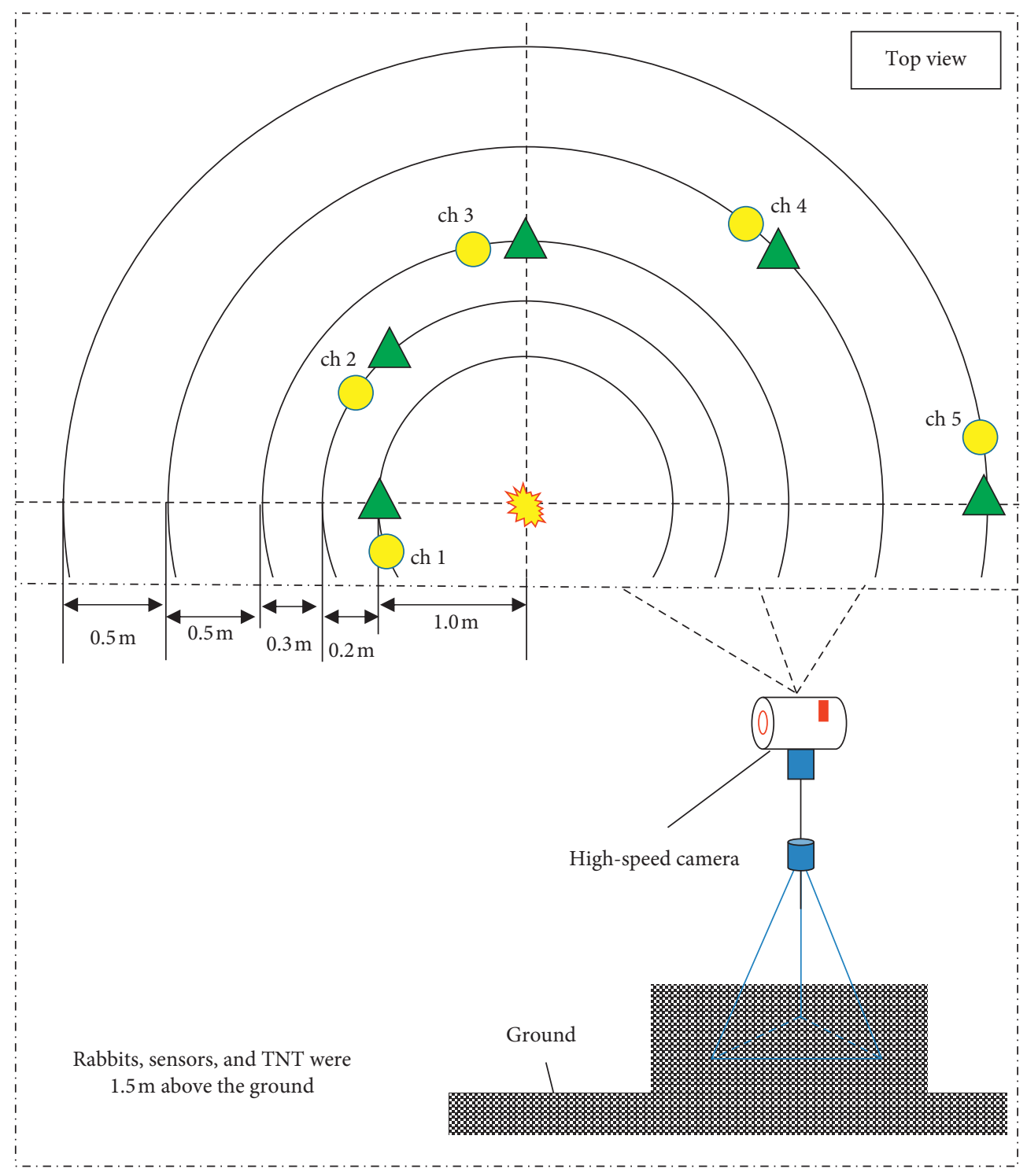

TNT blast source

Pressure sensor

Rabbit

FIGURE 1: Schematic diagram of experimental site layout.

The peak overpressures of the blast shock wave at different test points were obtained according to the empirical calculations and LS-DYNA numerical simulations as listed in Table 1. The empirical calculations and numerical simulation values are in close agreement. The formulas we used thus provided an accurate reference for the selection of experimental TNT equivalents and explosion distances.

3.2. Experimental Data on Shock Wave Overpressure. As discussed above, explosion experiments were carried out in six replications. The explosion process (from $\mathrm{P}_{1}$ to $\mathrm{P}_{9}$ ) was recorded by the high-speed camera as illustrated in
Supplementary Figure 3 . The interval between $\mathrm{P}_{1}$ and $\mathrm{P}_{2}$ was $1 \mathrm{~ms}$; the interval between $\mathrm{P}_{2}$ and $\mathrm{P}_{3}$ was $9 \mathrm{~ms}$. The interval between the photos shown below was $10 \mathrm{~ms}$. The relations between the shock wave overpressure and time at different positions are delineated in Figure 3.

Figures 3(a)-3(e) are overpressure-time curves of the shock waves at distances of $1.0,1.2,1.5,2.0$, and $2.5 \mathrm{~m}$, respectively. The curves measured by corresponding experiments were not as smooth as the curves obtained by numerical simulation. The experimental curves also had multiple peaks; the first peak was the target value of the experiment. The peak appeared earlier when it was closer to the explosion center, while subsequent peaks were formed by 


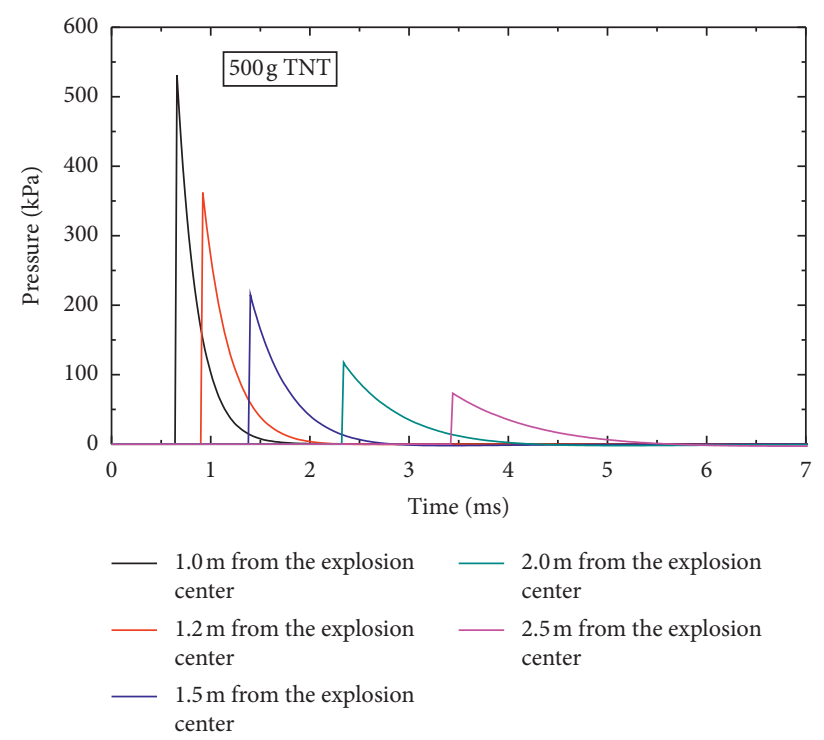

FIgURe 2: Overpressure-time curve of $500 \mathrm{~g}$ TNT (LS-DYNA).

TABLE 1: Peak overpressures at varying distance from $500 \mathrm{~g}$ TNT blast.

\begin{tabular}{ccccc}
\hline TNT quality $(w / g)$ & Distance $(r / \mathrm{m})$ & Contrast distance $\bar{r}=r / \sqrt[3]{w}$ & LS-DYNA & $\begin{array}{r}\text { Peak overpressure (kPa) } \\
\text { M. Aadovskyi's empirical formula }\end{array}$ \\
\hline & 1.0 & 1.26 & 559 & 546 \\
500 & 1.2 & 1.51 & 379 & 350 \\
& 1.5 & 1.89 & 226 & 208 \\
& 2.0 & 2.52 & 114 & 711 \\
\hline
\end{tabular}

shock wave reflection and other interference factors. The shapes of the overpressure-time curves are different at different locations, which reflects the complexity of the interference factors in shock wave overpressure measurement.

The peak overpressure of the blast wave at different propagation distances was obtained according to the information shown in Figure 3. The average values of the six repeated tests were taken as the final measured values, as reported in Table 2 .

For comparison among the measured, estimated, and simulated values of blast wave peak overpressure, three curves are plotted based on Tables 1 and 2, as also shown in Figure 4. In the beginning and the ending part of the curves, the experimental value was smaller than the empirical formula or numerical simulation values. In the middle part, the experimental value exceeded the other two. Generally speaking, the three curves were close enough to indicate sound agreement among the three sets of data.

3.3. Injury Situation of the Rabbits. The apparent medical damage to the rabbits' lung anatomies was evaluated after each explosion. The damage was observed with the naked eyes initially and later by HE staining to determine injury to the lung tissue at the cellular level under a scanning electron microscope (SEM). The HE staining images of normal lung tissue are illustrated in Figure 5 and those of blast-injured tissue are demonstrated in Figure 6.

The normal lung tissue is clear: The cell membrane was well connected and the space tissue was intact. There was neither obvious hyperemia or edema nor inflammatory cell infiltration or fibrosis. No obvious exudate was observed in the alveolar space. The alveolar structure was normal and without any obvious rupture.

The alveolar structure was destroyed and inflammatory cell infiltration occurred in all blast groups regardless of distance to the explosion. A large number of inflammatory cells exuded (black arrows) and filled the surrounding alveoli. The alveolar structure broke, expanded, and fused to form bullae of lung (red arrows) with marked bleeding and localized atelectasis. Injury to the lung was more severe, however, when nearer the explosive center. The 10X microscopy showed near-complete destruction of the alveoli structure at 1.0 and $1.2 \mathrm{~m}$ with only a few normal structural alveoli. The cells were compressed and there was considerable bleeding in this sample as well. An abundance of inflammatory cells were present in the $40 \mathrm{X}$ microscopy with some apoptotic cells at the $1.0 \mathrm{~m}$ distance. Alveolar damage and the extent of hemorrhaging decreased as distance to the blast increased. The 10X microscopy showed normal partial alveolar structures at $1.5,2.0$, and $2.5 \mathrm{~m}$, with relatively little bleeding. The $40 \mathrm{X}$ microscopy showed fewer inflammatory cells and no apoptotic cells at greater distance from the explosion center. 


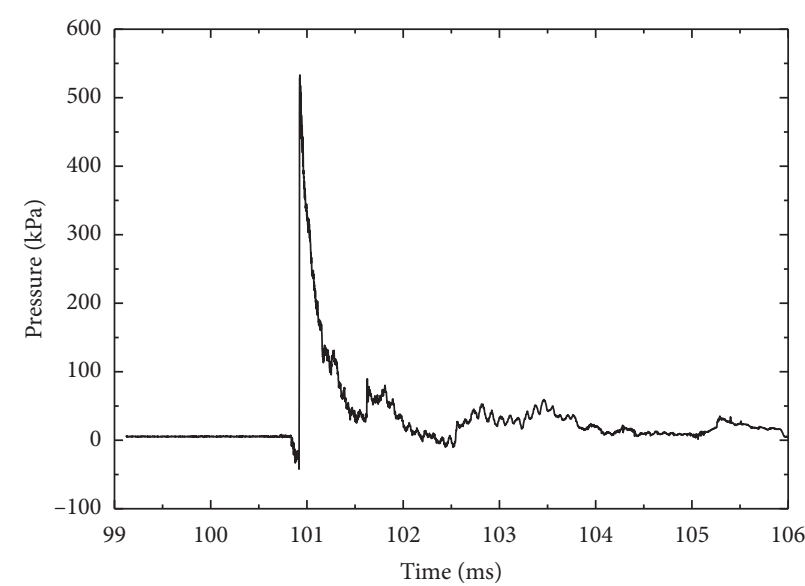

ch1: $p=533 \mathrm{kPa}$

(a)

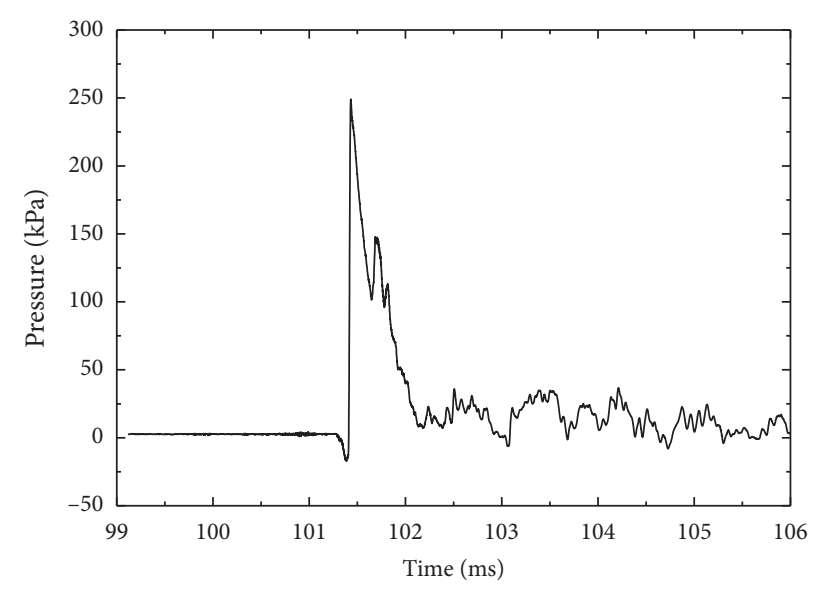

ch $3: p=249 \mathrm{kPa}$

(c)

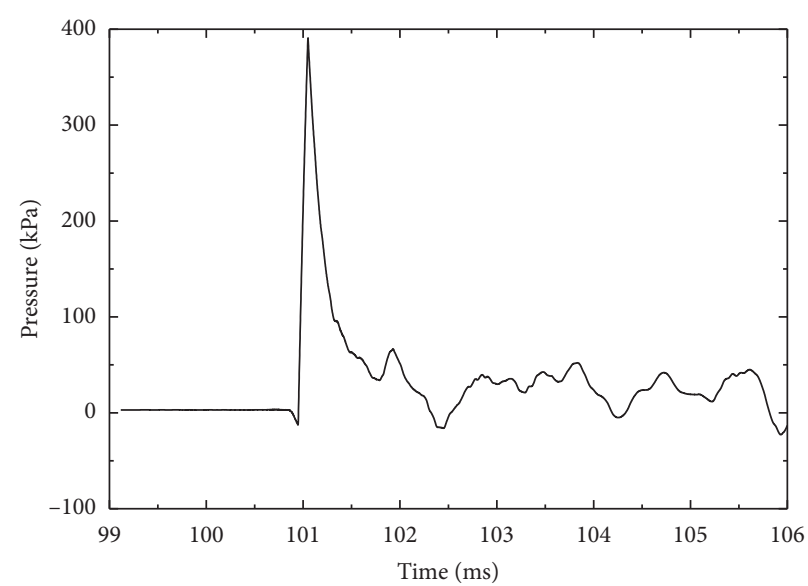

ch $2: p=390 \mathrm{kPa}$

(b)

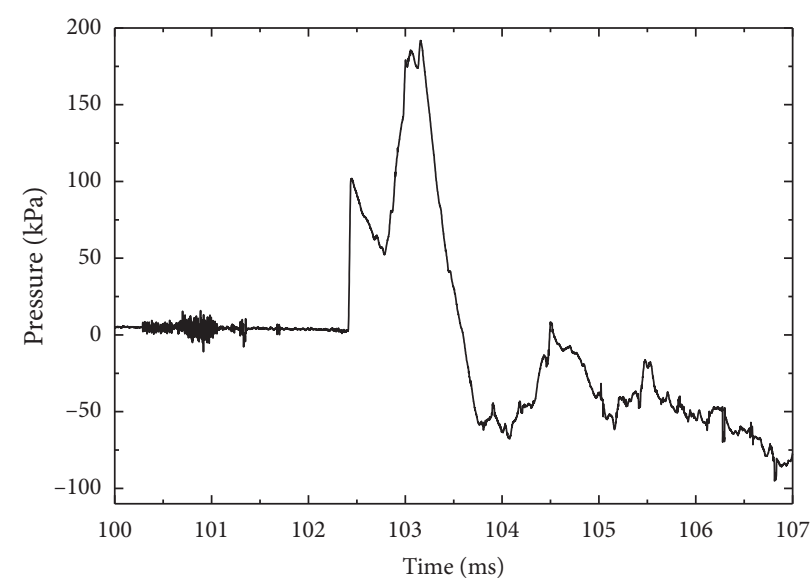

— ch4: $p=102 \mathrm{kPa}$

(d)

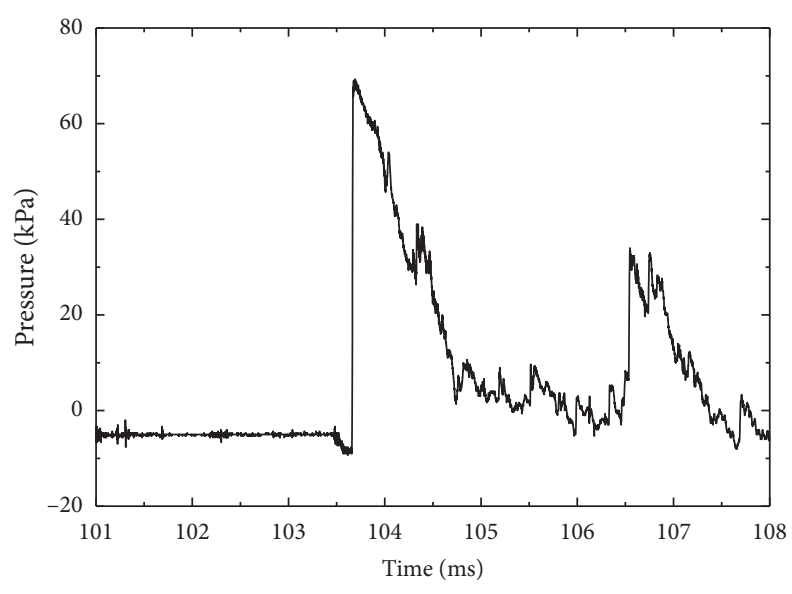

ch5: $p=69 \mathrm{kPa}$

(e)

FIGURE 3: Overpressure-time curve with different locations to explosion center. (a) $1.0 \mathrm{~m}$; (b) $1.2 \mathrm{~m}$; (c) $1.5 \mathrm{~m}$; (d) $2.0 \mathrm{~m}$; (e) $2.5 \mathrm{~m}$. 
TABLE 2: Peak overpressures of $500 \mathrm{~g}$ TNT blast.

\begin{tabular}{cccc}
\hline TNT quality $(w / g)$ & Distance $(r / \mathrm{m})$ & Contrast distance $\bar{r}=r / \sqrt[3]{w}$ & Peak overpressure $(\mathrm{kPa})$ \\
\hline \multirow{4}{*}{500} & 1.0 & 1.26 & 533 \\
& 1.2 & 1.51 & 390 \\
& 1.5 & 1.89 & 249 \\
& 2.0 & 2.52 & 102 \\
69 & 3.15 & 62 \\
\hline
\end{tabular}

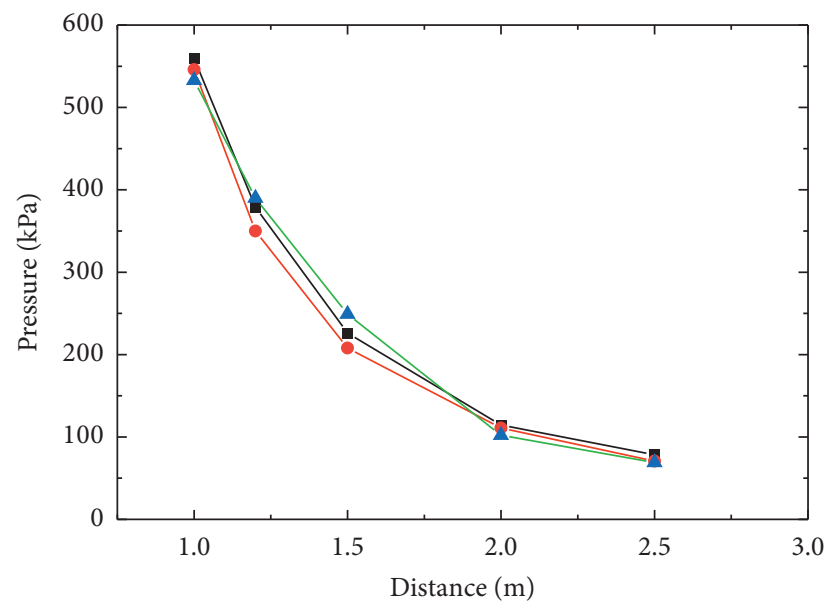

-- M.A. Sadovskyi's empirical formula
- - Experimental data
- - LS-DYNA

Figure 4: Comparison of experimental and estimated data.

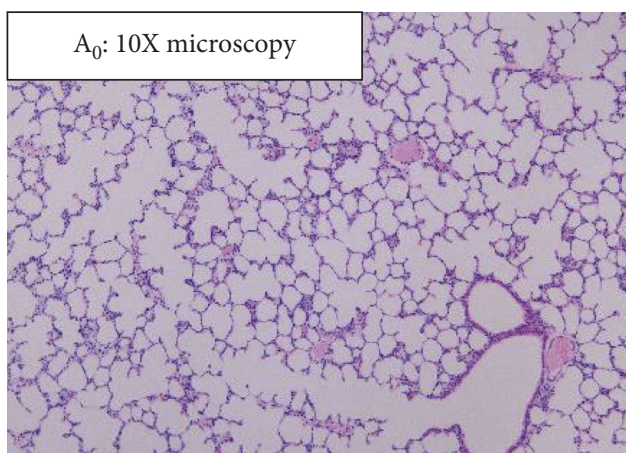

(a)

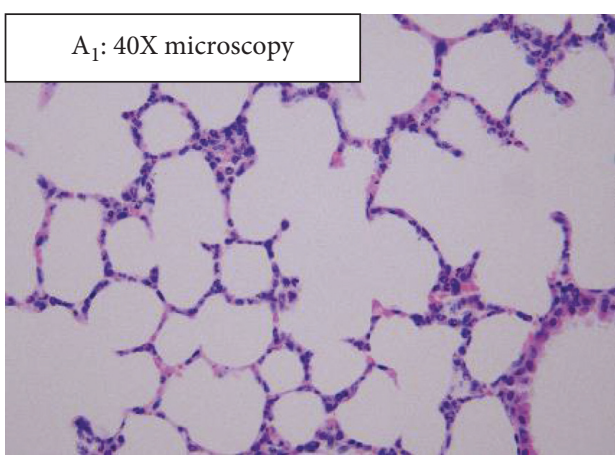

(b)

Figure 5: HE-stained lung tissue cells (control group).

\section{Discussion}

4.1. Comparative Analysis of Shock Wave Overpressure. As shown in Figures 2 and 3, the overpressure-time curves obtained by the experiments are not as smooth as the typical shock wave overpressure-time curves; there were numerous interruption signals which affected the accuracy in the actual test process. Blast shock wave tests are usually carried out with multifragmentation, strong vibration shock, transient high temperature, and other parameters. The test process is typically influenced by several factors. For example, the signal produces a spike in interference when the ballistic 

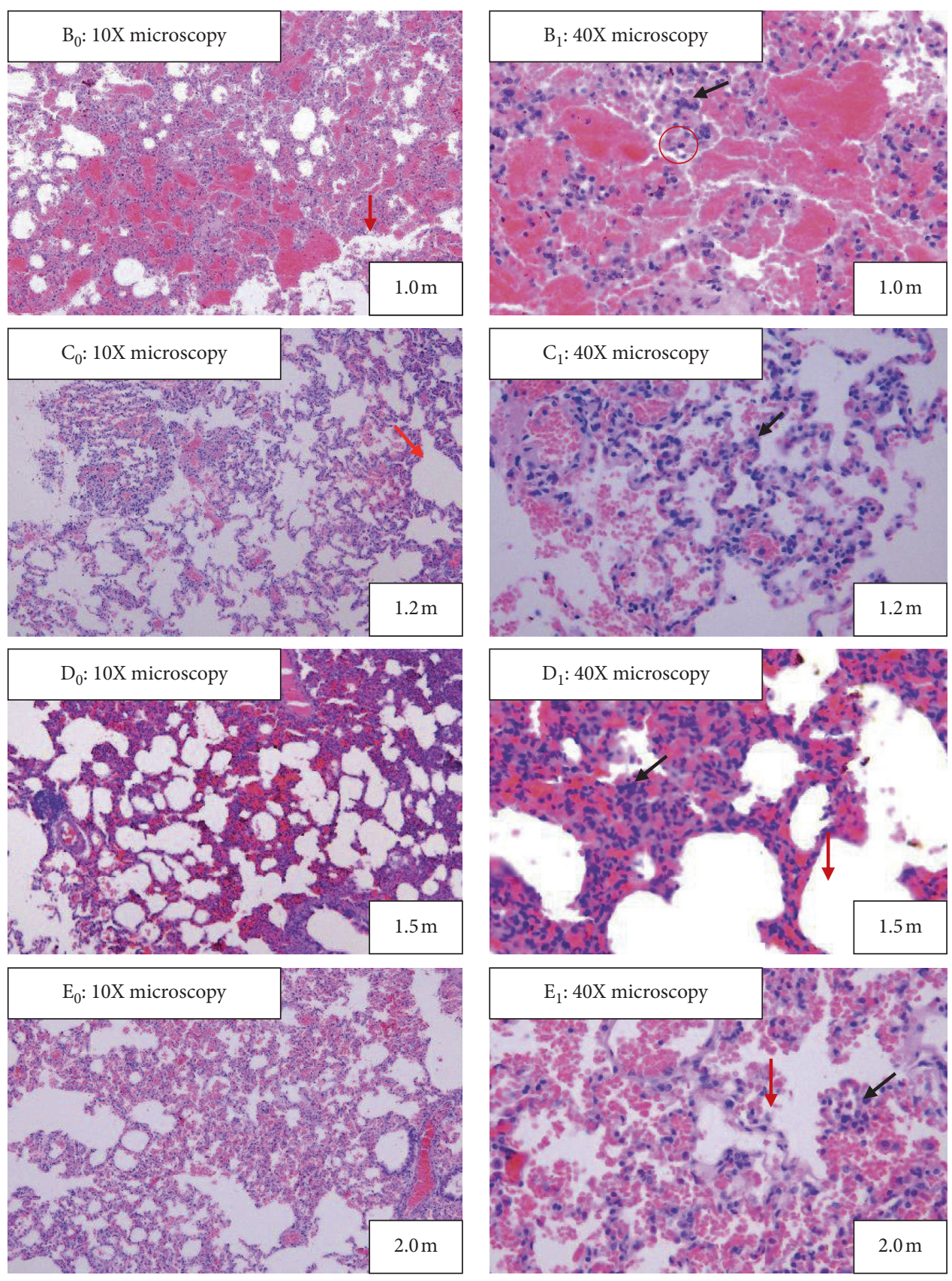

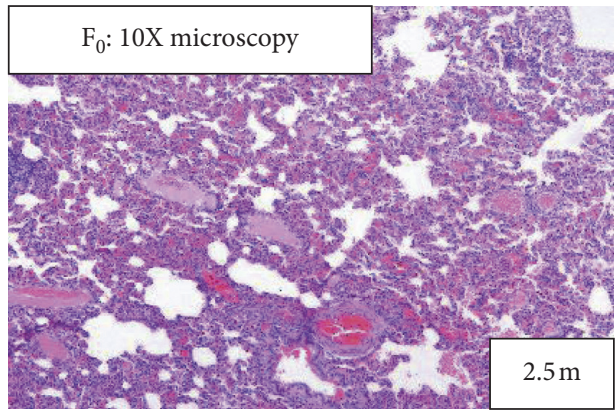

(a)

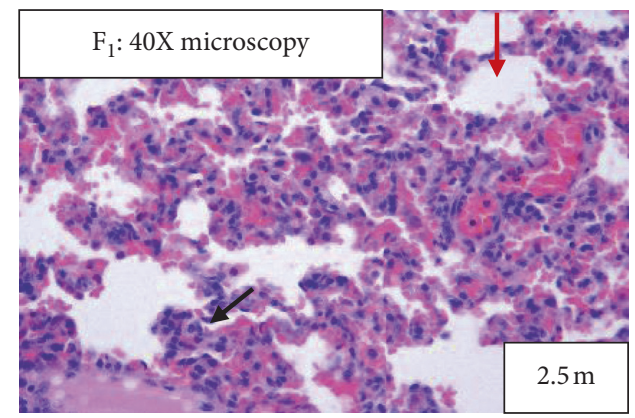

(b)

FIGURE 6: HE-stained photos of blast-injured lung tissue cells with varying distance to the explosion center. (a) 10X magnification and (b) 40X magnification. B, $1.0 \mathrm{~m}$ from explosion center; C, $1.2 \mathrm{~m} ; \mathrm{D}, 1.5 \mathrm{~m} ; \mathrm{E}, 2.0 \mathrm{~m} ; \mathrm{F}, 2.5 \mathrm{~m}$. 
TABLE 3: Injury of rabbits by blast shock wave.

\begin{tabular}{lcccc}
\hline No. & Distance $(r / \mathrm{m})$ & Peak overpressure $(\mathrm{kPa})$ & Injury level & Injury situation \\
\hline 1 & 1.0 & 533 & Severe & Alveolar structure completely destroyed; partial cell apoptosis \\
2 & 1.2 & 390 & Severe & Most alveolar structures destroyed \\
3 & 1.5 & 249 & Moderate & Moderate \\
4 & 2.0 & 102 & Mild & A small number of alveolar structures destroyed \\
5 & 2.5 & 69 & .
\end{tabular}

TABle 4: Traditional damage criteria of shock wave overpressure to human bodies (unit: $\mathrm{kPa}$ ).

\begin{tabular}{|c|c|c|c|c|}
\hline \multirow{2}{*}{ Data sources } & \multicolumn{4}{|c|}{ Injury level } \\
\hline & Mild & Moderate & Severe & Fatal \\
\hline American scholar & 15.7 & 23.5 & 53.9 & 186.34 \\
\hline Former soviet scholar & $19.6-39.2$ & - & $39.2-98.0$ & 235.4 \\
\hline Case statistics of explosion accident & $10.8-27.5$ & $27.5-49.0$ & $49.0-127.5$ & 127.5 \\
\hline
\end{tabular}

wave produced by the fragment passes through the sensor surface. The explosive test is usually accompanied by strong mechanical shocks, such as seismic waves or the mechanical impact of the sensor mounting plate. In addition, hightemperature effects cause the measured overpressure shock curve to drift. The explosion also produced high-speed charged ions which form electromagnetic waves acting on sensors and connecting cables to produce interference signals in the pressure test channels. There was also drift on the test signal due to the low detection frequency of the test system. In short, the entire test system was multiple-input and single-output. The signals of shock wave overpressure were mixed with plenty of complex interference signals. Accordingly, it was essential to filter the shock wave to secure accurate results.

As shown in Figure 4, the measured peak overpressure of the blast shock wave diverged from the empirical and simulated values; however, the differences were fairly slight. In effect, previously published empirical formulas did have reference significance for estimating explosion shock wave overpressure. The numerical simulation method we used was also applicable in the early stages of prediction. Differences between the measured and estimated values were attributable to the disturbances in the testing process. Further, the estimated value can only be used as a reference; the actual value must be based on a physical experiment. The working condition of the empirical formula was the explosion of TNT spherical charges in an infinite air medium, which was not fully replicable in an actual experiment. Generally speaking, however, the overpressure test results were in line with the calculations.

\subsection{Analysis between Damage Criterion and Experimental} Results. We divided the level of injury across our sample into four levels according to the degree of destruction of the alveolar structure: mild, moderate, severe, and fatal (Table 3).

As shown in Table 3, the shock wave overpressure in 69-102 kPa caused mild-to-moderate injury to the rabbit; the shock wave overpressure in $102-249 \mathrm{kPa}$ caused moderate injury; the shock wave overpressure in $249-390 \mathrm{kPa}$ resulted in moderate-to-severe injury; and the shock wave overpressure in $390-533 \mathrm{kPa}$ caused severe injury to the rabbit. The mild, moderate, and severe injuries, respectively, represented a small number of alveolar structures destroyed, most alveolar structures destroyed, and the alveolar structure completely destroyed accompanied by partial cell apoptosis.

Table 4 presents the traditional damage criteria of shock wave overpressure to the human body. The relationship between the shock wave overpressure and injury to rabbit lungs was far different from the data given by the traditional damage criterion, which was related to the posture of the rabbit in the experiment [22-24]. The rabbit's chest and abdomen faced the explosion center in our experiment and the lung was the target organ of the explosion shock wave. Therefore, the injury to our rabbits was rather severe. It was crucial to rely on not only extant blast wave damage criteria but also experimental data as a primary evaluation criterion when evaluating the effects of shock wave overpressure. In addition, the characteristics of the experimental scene and experimental conditions must also be properly considered. The existing shock wave damage criteria did not account for any experimental conditions and were not supported by clear-cut test parameters or methodology. Objectives and comprehensive evaluations should be defined according to the specific situation (e.g., target object and target scene were explosion mode) to accurately assess the damage effects of blast shock waves. The damage criteria of shock wave should not be referenced unilaterally; impulse criteria of shock waves should also be considered.

4.3. Mechanism of Lung Injury in Rabbits. As shown in Table 3, the rabbits suffered varying degrees of damage under the action of different blast shock wave overpressures. The mechanism of lung injury related to hemodynamics theory and to the pressure difference between the fluid and gas phases in the lung. When the air blast wave acted directly on the chest wall, the volume of gas in the thoracic cavity decreased sharply and swiftly spiked the local pressure in the thoracic cavity (tens-fold or even hundreds-fold). The negative pressure led to a prompt expansion on the compressed air bubbles in the lung, which teared the 
surrounding capillaries and venules, causing bleeding and allowing blood to enter the trachea. The mixture of edema fluid and blood formed a pulmonary edema. Injuries to rabbits, from mild to fatal, were characterized by the amount of bleeding and severity of arterial air embolism.

4.4. Injury Effects of Shock Wave Overpressure on Rabbits. The shock wave can be divided into three stages according to the damage caused. The first damage effect originated in the peak overpressure of the blast shock wave. The second damage effect involved driving penetration or nonpenetration of rock fragments and other explosion fragments. The third damage effect was caused by the entire displacement of the target due to the shock wave and pneumatic pressure. The influence of all three factors must be considered when evaluating the damage effects of blast waves on rabbits as opposed to simply the overpressure; the duration of the positive pressure zone also merited careful consideration, as well as the specific impulse of the shock wave.

\section{Conclusions}

Six repeated experiments were conducted in this study to delve into the effects of blast shock waves on rabbit lungs. We compared theoretical calculations and experimental data including preliminary observations of injuries, medical anatomy assessment, and postprocessing of HE staining. The conclusions can be summarized as follows:

(1) The blast shock wave overpressures estimated by numerical simulation and empirical formula were deemed accurate by comparison against the overpressure determined in the experiment.

(2) The shock wave created complex injuries in the rabbits characterized by interactions among multiple factors (e.g., dominant overpressure, fragments, and posture). The shock wave overpressure defined in this study was $69-533 \mathrm{kPa}$, which caused mild-tosevere injury to the rabbits. Mild, moderate, and severe injuries were defined, respectively, by a minute number of alveolar structures destroyed, most alveolar structures destroyed, and alveolar structure completely destroyed accompanied by partial cell apoptosis. Our results diverged considerably from those in the extant criteria for shock wave overpressure. To this effect, the results may be used to modify, complement, and perfect the criteria to enhance their precision and efficacy.

(3) The mechanism of lung injury was highly complicated and not yet exceptionally clear. Our results indicated that lung injury in rabbits was caused by pulmonary hemorrhage and pulmonary edema.

\section{Data Availability}

The data used to support the findings of this study are included within the article.

\section{Conflicts of Interest}

The authors declare that there are no conflicts of interest regarding the publication of this paper.

\section{Acknowledgments}

This work was financially supported by the National Key Research and Development Program of China (Grant no. 2017YFC0804700).

\section{Supplementary Materials}

Supplementary Figure 1: experimental site layout. Supplementary Figure 2: schematic diagram of test equipment connection. Supplementary Figure 3: pictures (P1 to P9) on explosion of $500 \mathrm{~g}$ TNT. (Supplementary Materials)

\section{References}

[1] J. G. Owens, "Physical therapy of the patient with foot and ankle injuries sustained in combat," Foot and Ankle Clinics, vol. 15, no. 1, pp. 175-186, 2010.

[2] H. R. Champion, J. B. Holcomb, and L. A. Young, "Injuries from explosions: physics, biophysics, pathology, and required research focus," The Journal of Trauma: Injury, Infection, and Critical Care, vol. 66, no. 5, pp. 1468-1477, 2009.

[3] A. J. H. Korver, "Injuries of the lower limbs caused by antipersonnel mines: the experience of the International Committee of the Red Cross," Injury, vol. 27, no. 7, pp. 477-479, 1996.

[4] J. Ning, L. Mo, H. Zhao et al., "Sodium hydrosulphide alleviates remote lung injury following limb traumatic injury in rats," PLoS One, vol. 8, no. 3, Article ID e59100, 2013.

[5] T. Josey, Investigation of Blast Load Characteristics On Lung Injury, University of Waterloo, Waterloo, Canada, 2010.

[6] P. Singer, C. JD, and M. Stein, "Conventional terrorism and critical care," Critical Care Medicine, vol. 33, no. 1 Suppl, p. S61, 2005.

[7] Q. Zhang, X. M. Qian, Y. Y. Chen, and M. Yuan, "Deflagration shock wave dynamics of DME/LPG blended clean fuel under the coupling effect of initial pressure and equivalence ratio in elongated closed space," Journal of Cleaner Production, vol. 250, Article ID 119572, 2019.

[8] J. K. Zhang, Z. G. Wang, H. G. Leng, and Z. Yang, "Studies on lung injuries caused by blast underpressure," The Journal of Trauma and Acute Care Surgery, vol. 40, no. 3 Suppl, pp. 77-80, 1996.

[9] A. Barnett-Vanes, A. Sharrock, T. Eftaxiopoulou et al., "CD43Lo classical monocytes participate in the cellular immune response to isolated primary blast lung injury," Journal of Trauma and Acute Care Surgery, vol. 81, no. 3, pp. 500-511, 2016.

[10] A. E. Ritenour and T. W. Baskin, "Primary blast injury: update on diagnosis and treatment," Critical Care Medicine, vol. 36, no. 7 Suppl, pp. 311-317, 2008.

[11] E. R. Frykberg and J. J. Tepas, "Terrorist bombings. lessons learned from belfast to beirut," Annals of Surgery, vol. 208, no. 5, pp. 569-576, 1988.

[12] M. Aboudara, P. F. Mahoney, B. Hicks, and D. Cuadrado, "Primary blast lung injury at a NATO role 3 hospital," Journal of the Royal Army Medical Corps, vol. 160, no. 2, pp. 161-166, 2014. 
[13] M. Aboudara, B. Hicks, D. Cuadrado, P. F. Mahoney, and J. Docekal, "Impact of primary blast lung injury during combat operations in Afghanistan," Journal of the Royal Army Medical Corps, vol. 162, no. 1, p. 75, 2016.

[14] J. Z. Huang, Z. Yang, Z. Wang, and H. Leng, "Study on characteristics of blast-fragment combined injury in dogs," The Journal of Trauma and Acute Care Surgery, vol. 40, no. 3 Suppl, pp. 63-67, 1996.

[15] Z. H. Yang, Z. G. Wang, C. G. Tang, and Y. Ying, "Biological effects of weak blast waves and safety limits for internal organ injury in the human body," The Journal of Trauma and Acute Care Surgery, vol. 40, no. 3 Suppl, pp. 81-84, 1996.

[16] J. Q. Fan, H. X. Dong, Y. H. Gao et al., "Experimental investigation on damage effects on animals under the action of blast wave in a chamber," Journal of Vibration and Shock, vol. 32, no. 9, pp. 35-39, 2013.

[17] H. B. Chen, Z. G. Wang, Z. H. Yang et al., "Injury of animal lungs in the experiments to simulate the three phases of shock wave propagation," Explosion and Shock Waves, vol. 20, no. 3, pp. 264-269, 2000.

[18] Z. H. Yang, X. Y. Li, X. Ning et al., "A comparative study on viscera injuries caused by underwater blast wave and air blast wave," Chinese Journal of Nautical Medicine and Hyperbaric Medicine, vol. 13, no. 2, pp. 65-68, 2006.

[19] T. Josey, D. Cronin, C. P. Salisbury, and K. V. Williams, "The effect of blast load conditions on lung injury," Journal of Biomechanics, vol. 39, no. 39, p. S163, 2006.

[20] E. R. Frykberg, Explosions and Blast Injury, Essentials of Terror Medicine, Springer, New York, NY, USA, 2009.

[21] J. Henrych, The Dynamics of Explosion and Its Use, Elsevier/ North Holland, New York, NY, USA, 1979.

[22] P. F. Acosta, "Overview of UFC 3-340-02 structures to resist the effects of accidental explosions," in Proceedings of the Structures Congress, pp. 1454-1469, Las Vegas, NV, USA, April 2011.

[23] F. X. Cao, Study on Multi-Damage Effect of Explosion, Nanjing University of Science and Technology, Nanjing, China, 2008.

[24] Z. Li, "Safety distance for persons under blast air shock," Explosion and Shock Waves, vol. 10, no. 2, pp. 135-144, 1990. 